\title{
A função da MnSOD Ala16Val, O stress oxidativo e marcadores de apoptose como biomarcadores em pacientes com Acidente Vascular Encefálico Crônico
}

\author{
Eduardo Tanuri Pascotini ${ }^{1}$; ${ }^{2}$ ascotini E.T.;
}

\section{Resumo}

O Acidente Vascular Encefálico (AVE) é uma das principais causas de morte e incapacidade, sendo considerada a segunda maior causa de mortes no mundo.Entre todos os países da América Latina, o Brasil é o que apresenta as maiores taxas de mortalidade por AVE. Sobreviventes de AVE podem sofrer várias sequelas como hemiparesias, desordens na comunicação e déficit cognitivo, as quais tem um impacto importante na vida dos pacientes e custos consideráveis para a saúde. Essas alterações clínicas estão relacionadas com o dano cerebral, o qual pode ser causado pelo stress oxidativo decorrente da falha da enzima Manganês Superóxido Dismutase (MnSOD). Essa enzima, localizada na mitocôndria, é capaz de transformar o radical superóxido (O2-) em produto não tóxico para a célula. A SOD é codificada por um único gene que apresenta um polimorfismo comum o qual resulta na substituição do aminoácido alanina (A), por uma valina (V) no códon 16 do DNA. Estudos anteriores descreveram uma associação entre o genótipo VV e o acometimento de pacientes com doenças neurológicas, incluindo o Acidente Vascular Cerebral. Diante disso, o presente estudo tem o objetivo de investigar a associação dos biomarcadores de apoptose e stress oxidativo com o polimorfismo da SOD nos pacientes pós-AVE comparando com controles.

Palavras Chave: SOD; polimorfismo; AVE.

\footnotetext{
1 Autor .Doutorando de Farmacologia da Universidade Federal de Santa Maria; Pesquisa financiada pela CAPES, CNPQ. E-mail:dudatanuri@hotmail.com

*Apresentadora e co-autora. Graduanda de Medicina da Universidade Federal de Santa Maria. Bolsista CAPES. E-mail: bele-92@hotmail.com
} 


\section{Método e Resultados}

A pesquisa foi realizada com os pacientes do ambulatório de neurologia do Hospital Universitário de Santa Maria (HUSM). Foi feito um estudo transversal com 40 pacientes com AVE crônico e 40 controles saudáveis, sendo $27(67,5 \%)$ do sexo masculino, com idade média de 60,5 anos. Em relação ao hemisfério afetado, 13 $(32,5 \%)$ pacientes sofreram AVE no hemisfério esquerdo e 27 no hemisfério direito (67,5\%). Nenhum paciente tem tempo de AVE de menos de 6 meses, 7 (17,5\%) tem AVE de seis a doze meses, $8(20 \%)$ tem de doze a vinte e quatro meses e $25(62,5 \%)$ tem tempo de AVE de mais de vinte e quatro meses. Em relação à pressão arterial, 17 $(42,5 \%)$ pacientes tem pressão arterial normal $(<130 / 85 \mathrm{mmHg}), 3(7,5 \%)$ tem pressão limítrofe $(130 / 85 \mathrm{mmHg}-139 / 89 \mathrm{mmHg})$ e $20 \quad(50 \%)$ tem hipertensão leve (140/90mmHg - 160/100mmHg). Nenhum paciente tem hipertensão moderada ou grave. Todos os pacientes (100\%) tem frequência cardíaca em repouso menor que 100bpm. Foi coletada uma amostra de sangue para posterior determinação do genótipo e níveis séricos da proteína carbonil, caspases 1,3 e 8, as quais são marcadoras de stress oxidativo e apoptose. Os pacientes com AVE apresentaram alta frequência do genótipo VV (40\%) comparados ao grupo controle (10\%). Os níveis séricos da proteína carbonil $(\mathrm{t}=7,5 \mathrm{nmol} / \mathrm{mg})$, caspase $1(\mathrm{t}=6,9 \mathrm{U} / \mathrm{g})$, caspase $3(\mathrm{t}=6,8 \mathrm{U} / \mathrm{g})$ e caspase $8 \quad(\mathrm{t}=6,7 \mathrm{U} / \mathrm{g})$ estavam aumentados nos pacientes com AVE comparados aos controles.

\section{Discussão e conclusões}

A inflamação tem um papel significativo na patogênese do AVE. A resposta inflamatória é caracterizada pela produção e liberação de citocinas pró-inflamatórias, como o TNF- $\alpha$, IL-1, IL-6 e IL-18, pela ativação de células incluindo neurônios, astrócitos, micróglia e células endoteliais resultando na morte de células gliais e neuronais durante a isquemia cerebral (David et al., 2012). A liberação de TNF- $\alpha$ pode levar a ativação de seu receptor cuja interação com o ligante de Fas pode levar a ativação da caspase 8 pela via intrínseca ou pela ativação de proteínas pró-apoptóticas que liberam o citocromo $\mathrm{c}$ da membrana mitocondrial o qual ativa a caspase 9 pela via extrínseca resultando em morte celular programada, consequentemente levando à lesão do DNA (Yazdi et al., 2010).

Nosso estudo, então, sugere que o genótipo VV pode influenciar no aumento do stress oxidativo, resultando no aumento dos marcadores apoptóticos em pacientes com AVE crônico. Paciente com esse genótipo poderiam ter mais chances de novo AVE. Além disso, os resultados encontrados sugerem que o dano cerebral pós-AVE pode estar associado às vias apoptóticas e morte celular, representado pelo aumento dos níveis das caspases 1,3 e 8 em relação aos controles. 


\section{Tabela e figuras}

Figura 1: Valor da proteína carbonil no grupo controle e no grupo com AVE. ${ }^{*}(p<0,05)$

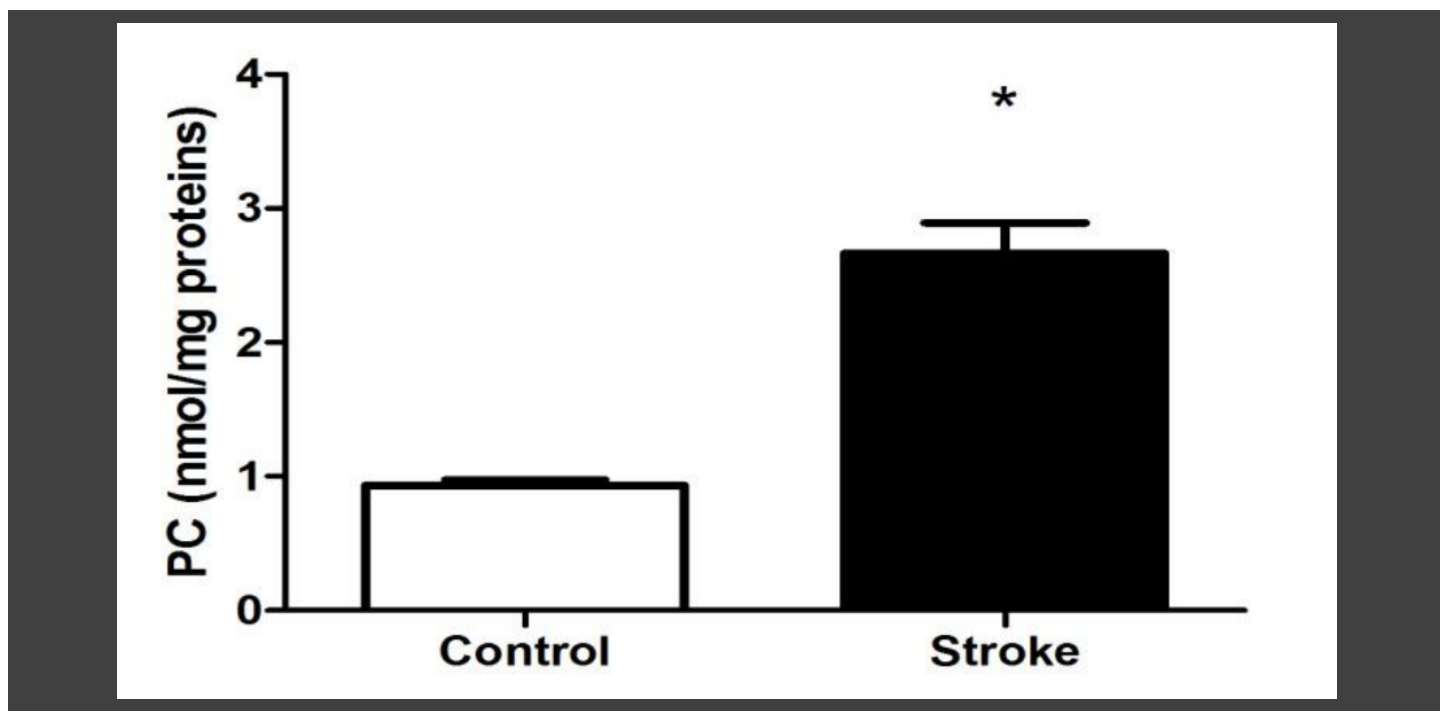

Fonte: Figura feita no departamento de Neuropsiquiatria da Universidade Federal de Santa Maria (UFSM).

Figura 2: Valor da caspase 1 no grupo controle e no grupo com AVE. ${ }^{*}(p<0,05)$

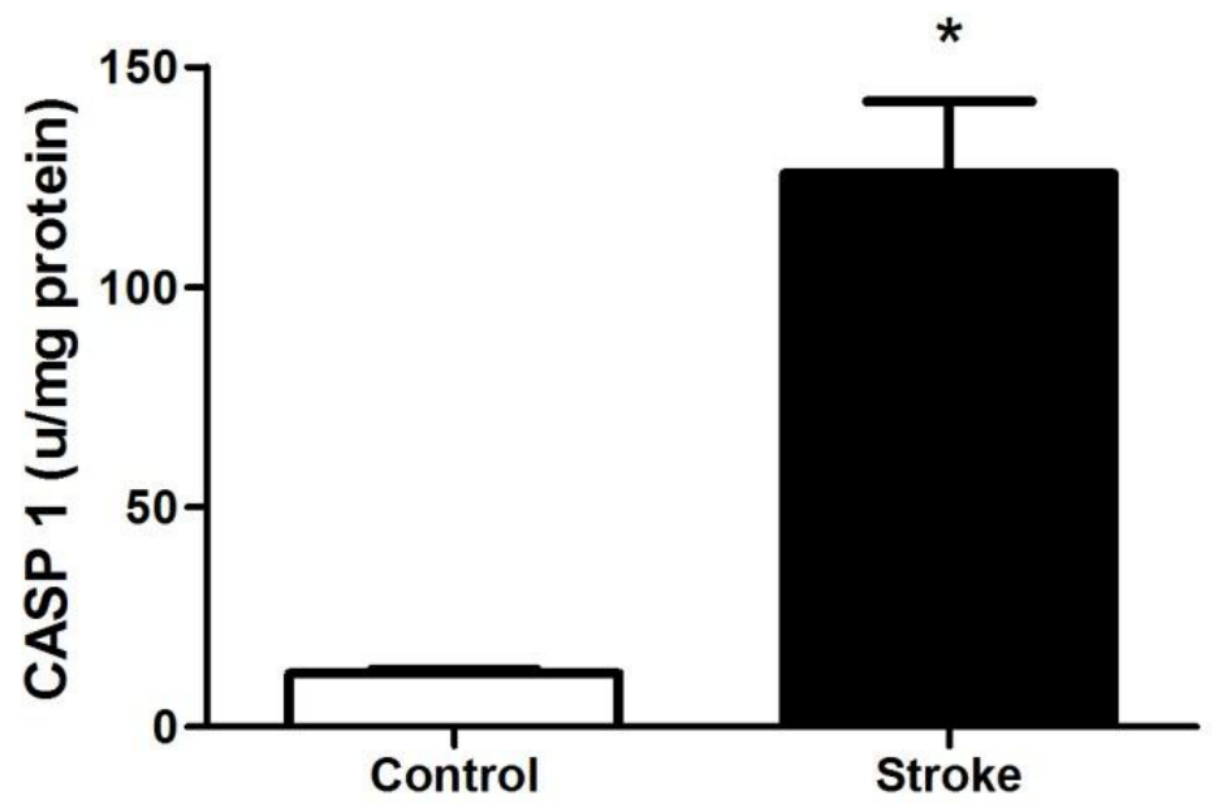

Fonte: Figura feita no departamento de Neuropsiquiatria da Universidade Federal de Santa Maria (UFSM). 
Figura 3: Valores da caspase 3 (A) e caspase 8 (B) no grupo controle e nos com AVE. ${ }^{*}(\mathrm{p}<0,05)$

A

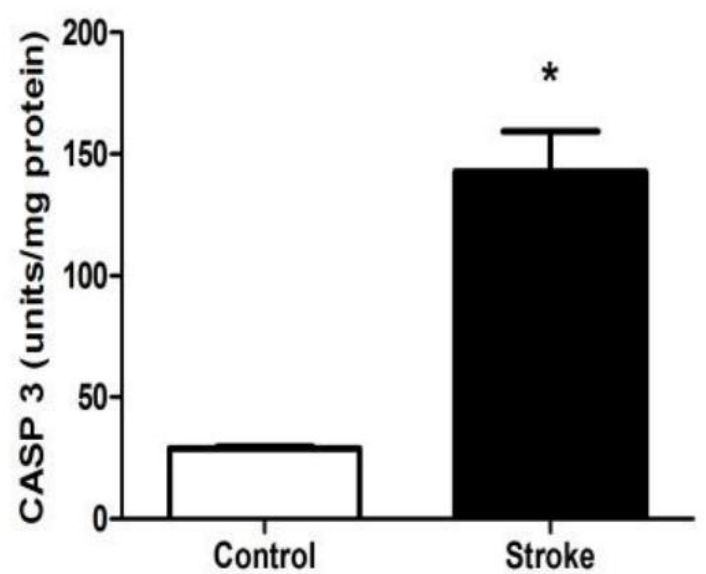

B

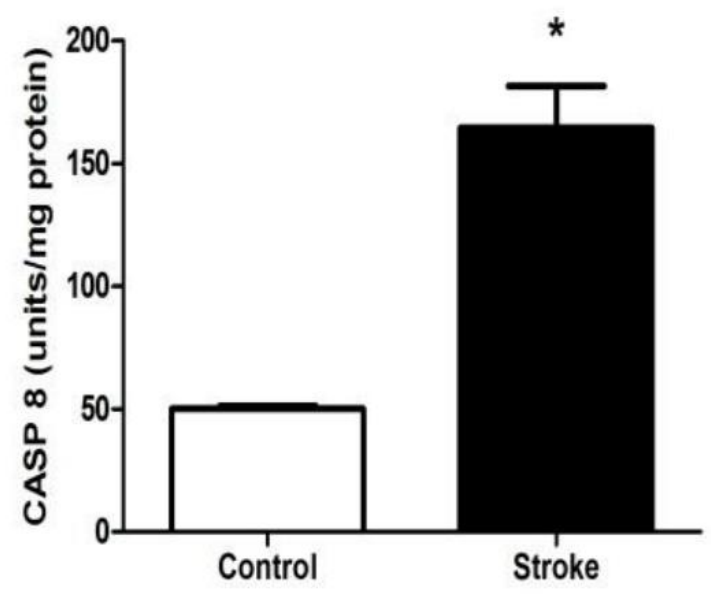

Fonte: Figura feita no departamento de Neuropsiquiatria da Universidade Federal de Santa Maria (UFSM).

\section{Citações}

\subsubsection{Indiretas}

A inflamação tem um papel significativo na patogênese do AVE. A resposta inflamatória é caracterizada pela produção e liberação de citocinas pró-inflamatórias, como o TNF- $\alpha$, IL-1, IL-6 e IL-18, pela ativação de células incluindo neurônios, astrócitos, micróglia e células endoteliais resultando na morte de células gliais e neuronais durante a isquemia cerebral (David et al., 2012).

A liberação de TNF- $\alpha$ pode levar a ativação de seu receptor cuja interação com o ligante de Fas pode levar a ativação da caspase 8 pela via intrínseca ou pela ativação de proteínas pró-apoptóticas que liberam o citocromo $c$ da membrana mitocondrial o qual ativa a caspase 9 pela via extrínseca resultando em morte celular programada, consequentemente levando à lesão do DNA (Yazdi et al., 2010). 


\title{
The functional of MnSOD Ala16Val, the oxidative stress and markers of apoptosis as biomarkers in patients with stroke
}

\begin{abstract}
The superoxide dismutase manganese dependent (MnSOD2) catalyzes O2 in $\mathrm{H} 2 \mathrm{O} 2$ into mitochondria and is encoded by a single gene that presents a common polymorphism that results in the replacement of alanine (A) with a valine $(V)$ in the 16 codon. This polymorphism has been implicated in a decreased efficiency of SOD2 transport into targeted mitochondria in $V$ allele carriers. Previous studies described an association between VV genotype and neurological diseases, including stroke. However, the causal mechanisms to explain this association need to be more elucidated. We postulated that the polymorphism could influence the inflammatory response. Then, we objective will be investigate if have a relationship in the inflammation biomarkers in the patients with chronic stroke.
\end{abstract}

Keywords: SOD2. polymorphism. chronic stroke. 


\section{Referências}

BOATRIGHT K.M, RENATUS M., SCOTT F.L., SPERANDIO S., SHIN H., PEDERSEN I.M, RICCI JE, EDRIS W.A., SUTHERLIN D.P., GREEN D.R, SALVESEN GS. A unified model for apical caspase activation. Mol. Cell. 2003; 11:529-541.

CHOWDURY I, THARAKAN B, BHAT GK. Caspases - na update. Comp Biochem Physiol B Biochem Mol Biol 2008; 151:10-27

DAVID YANG FANNA B., SEUNG-YOON L., SILVIA M., PRASAD G., CHRISTOPHER G. S., THIRUNA V. A. Pathogenesis of acute stroke and the role of inflammasomes. Ageing Research Reviews 2012; 12:941- 966

MARTINON F, MAYOR A, TSCHOPP J. The inflammasomes: guardians of the body. Annu. Rev. Immunol. 2009; 27:229-265

MUZIO M, CHINNAYAN AM, KISCHKEL FC, O'Rourke K, SHEVCHENKO A, Ni J, SCAFITTI C, BRETZ JD, ZHANG M, GENTZ R, MANN M., KRAMMER P.H, PETER M.E, DIXIT V.M. FLICE, a novel FADD - homologous ICE/CED - 3- like protease, is recruited to the CD95 (Fas/APO-1) death-inducing signaling complex. Cell 1996; 85:817-827

WOODRUFF, T.M., THUNDYL, J., TANG, S.C., SOBEY, C.G., TAYLOR, S.M., ARUMUGAN, T.V. Pathophysiology, treatment, and animal and cellular models of humanischemic stroke. Mol. Neurodegener. 2011; 6, 11-29.

YAZDI A.S, GUARDA G, D'OMBRAIN M.C, DREXLER S.K. Inflammatory Caspases in Innate Immunity and Inflammation. Journal of Innate Immunity. 2010; 2:228-237. 